\title{
An evaluation of the correlation between open solar flux and total solar irradiance
}

\begin{abstract}
M. Lockwood*
Rutherford Appleton Laboratory, Chilton, Didcot, Oxfordshire, UK

Department of Physics and Astronomy, University of Southampton, Southampton, Hampshire, UK

Received 1 October 2001 / Accepted 22 November 2001

Abstract. The correlation between the coronal source flux $F_{\mathrm{S}}$ and the total solar irradiance $I_{\mathrm{TS}}$ is re-evaluated in the light of an additional 5 years' data from the rising phase of solar cycle 23 and also by using cosmic ray fluxes detected at Earth. Tests on monthly averages show that the correlation with $F_{\mathrm{S}}$ deduced from the interplanetary magnetic field (correlation coefficient, $r=0.62$ ) is highly significant $(99.999 \%)$, but that there is insufficient data for the higher correlation with annual means $(r=0.80)$ to be considered significant. Anti-correlations between $I_{\mathrm{TS}}$ and cosmic ray fluxes are found in monthly data for all stations and geomagnetic rigidity cut-offs $(r$ ranging from -0.63 to -0.74 ) and these have significance levels between $85 \%$ and $98 \%$. In all cases, the fit is poorest for the earliest data (i.e., prior to 1982). Excluding these data improves the anticorrelation with cosmic rays to $r=-0.93$ for one-year running means. Both the interplanetary magnetic field data and the cosmic ray fluxes indicate that the total solar irradiance lags behind the open solar flux with a delay that is estimated to have an optimum value of 2.8 months (and is within the uncertainty range $0.8-8.0$ months at the $90 \%$ level).
\end{abstract}

Key words. Sun: magnetic fields - fundamental parameters - solar-terrestrial relations - interplanetary medium

\section{Introduction}

The total solar irradiance $I_{\mathrm{TS}}$ shows a solar cycle variation (Willson 1997; Fröhlich \& Lean 1998a, 1998b). Lockwood \& Stamper (1999) reported a statistical correlation between this variation and that in the coronal source flux $F_{\mathrm{S}}$ - the "open" magnetic flux of the Sun that threads the coronal source surface and is dragged into the heliosphere by the solar wind. These authors used annual mean data for 1981-1995 and produced fits of $I_{\mathrm{TS}}$ with $F_{\mathrm{S}}$ for data from the various irradiance monitors separately. The linear regressions were then used to inter-calibrate the data from the different instruments and an overall correlation coefficient of correlation coefficient, $r$, of 0.852 was obtained for the best-fit linear regression:

$\left[I_{\mathrm{TS}}\right.$ in $\left.\mathrm{W} \mathrm{m}^{-2}\right]=1364.9+(0.507)\left[F_{\mathrm{S}}\right.$ in $\left.10^{14} \mathrm{~Wb}\right]$.

The instruments used were the Hickey-Frieden (HF) radiometer on the Nimbus 7 satellite, ACRIM1 on Solar Maximum Mission (SMM), ERBS (Earth Radiation Budget Satellite), and ACRIM2 on the Upper Atmosphere Research Satellite (UARS). Subsequently, irradiance measurements have been made by the DIARAD and PMO6-V absolute radiometers of the VIRGO experiment on the SoHO spacecraft and inter-calibration of the data into a composite data series has been carried out by Fröhlich \& Lean (1998a, 1998b) and Fröhlich (2000).

* e-mail: M.Lockwood@rl.ac.uk
Wang et al. (2000a) confirmed that a correlation between $I_{\mathrm{TS}}$ and $F_{\mathrm{S}}$ is indeed present but noted that it is considerably weaker for monthly data and that the correlation is poor for the earliest data (before 1982).

Were it to reveal a real physical connection between $I_{\mathrm{TS}}$ and $F_{\mathrm{S}}$, this correlation would be very important even though it is unlikely to be the result of a direct causal relationship. The coronal source flux has been estimated from a sequence of geomagnetic observations that extends back to 1868 (Lockwood et al. 1999a): a physical link would mean that the correlation applies on century as well as decadal timescales, and so would allow us to use these $F_{\mathrm{S}}$ data to make a definitive reconstruction of the long-term irradiance variation. Using the correlation to make a simple extrapolation based on the one parameter, $F_{\mathrm{S}}$, Lockwood \& Stamper (1999) generated an irradiance reconstruction that was remarkably similar to others by Hoyt \& Schatten (1993), Solanki \& Fligge $(1998,1999)$, Lean et al. (1995) and, in particular, by Lean (2000). In these other cases, a long-term drift was superposed on an 11-year variation associated with the sunspot number, $R$. In order to quantify this long-term drift, Hoyt and Schatten used solar cycle length $L$, whereas Lean et al., Lean and Solanki and Fligge used the 11-year smoothed sunspot number, $R_{11}$. Lockwood (2001) has pointed out that there is a strong correlation between the century-scale variations of $F_{\mathrm{S}}, L$ and $R_{11}$ and thus it is not surprising that these reconstructions are similar in form. However, it 
is surprising that the reconstructions also give similar amplitudes of the long-term drift: for example, Lean et al. and Lean quantify this drift by comparison of non-cyclic stars with the Maunder-minimum Sun. One inference is that not only is the correlation between $I_{\mathrm{TS}}$ and $F_{\mathrm{S}}$ real, but that it applies on both 100-year and solar cycle timescales.

Furthermore, the rate of production of the ${ }^{10} \mathrm{Be}$ and ${ }^{14} \mathrm{C}$ isotopes, produced by cosmic ray bombardment of Earth's atmosphere, are also strongly anticorrelated with the reconstructed irradiance (Lean et al. 1995). Given that cosmic ray fluxes are also strongly anticorrelated with the heliospheric field that shields the inner heliosphere (Cane et al. 1999; Lockwood 2001), this also points to an underlying physical connection between irradiance and the open solar flux.

Such a connection is, in many ways, surprising. Irradiance variations on decadal (and possibly century) time scales are almost entirely due to flux tubes of strong magnetic field threading the solar photosphere. For flux tubes of radius exceeding about $250 \mathrm{~km}$, the main effect is blocking of upward heat flux to give sunspots, characterised by associated reduction in surface temperature and radiated power. Most of the blocked heat flux is returned to the convection zone with its huge thermal capacity and only a small fraction reaches the surface around the spots to give the low-contrast "bright ring" (Spruit 1982, 1991). Observations of these bright rings around isolated sunspots imply that they are of order $10 \mathrm{~K}$ hotter than the quiet photosphere and account for about $10 \%$ of the blocked heat flux (Rast et al. 1999). For flux tubes with smaller radius (less than about $250 \mathrm{~km}$ ) the upward heat flux is again blocked by the magnetic field but the temperature is nevertheless maintained by radiation from the flux tube walls. These smaller tubes are called faculae and particle concentration (and thus pressure) is lower within them because of the increased magnetic pressure. Thus the depth of a contour of constant optical depth is increased in the small flux tube and the temperature at that optical depth is also increased, giving enhanced emitted power. To an observer, this effect is strongest for faculae near the limb, because there the bright walls of the flux tube are most visible. The effect of individual faculae is much smaller than that of spots (contrasts are of order 1.01-1.1, depending on their location on the disk, whereas averaging umbrae and penumbrae yield that the contrast is of order 0.3 for sunspots); however, the fraction of the disk covered by faculae is roughly an order of magnitude greater than for sunspots. The total effect of facular brightening is an increase in $I_{\mathrm{TS}}$ of order $3 \mathrm{~W} \mathrm{~m}^{-2}$ at sunspot maximum compared to sunspot minimum, whereas sunspot darkening causes a decrease of order $1 \mathrm{~W} \mathrm{~m}^{-2}$ (Fröhlich \& Lean 1998a, 1998b). Computation of the irradiance can be made from surface magnetograms by characterising every element of the solar disk as either quiet sun, sunspot or faculae (e.g., Fligge et al. 1998). The results are an excellent match to the observed irradiance, on both the solar-cycle and solar rotation timescales (the former due to the variation of the total magnetic field threading the photosphere, the latter because individual features rotate across the disk).

However, the open magnetic flux that threads the coronal source surface is, at most, a few percent of the total flux threading the photosphere: most of the flux that has emerged through the photosphere (Harvey \& Zwaan 1993) closes in loops in the corona below the source surface (Wang et al. 2000a, 2000b). Furthermore, the dependence of contrast on flux tube radius means that how the photospheric flux is distributed spatially is also crucial to the net effect on $I_{\mathrm{TS}}$. The correlation of the open flux with irradiance could mean that the total open flux remains a relatively constant fraction of the total photospheric flux over the solar cycle, and that the distribution of flux tube sizes is also relatively fixed (Chapman et al. 1997). Alternatively, if there is a significant solar cycle variation in either one of these, then it must somehow be largely compensated for by changes in the other.

Thus it becomes important to check the validity and significance of the correlation reported by Lockwood \& Stamper (1999). In the present paper, we use the composite data series on total solar irradiance, including the recent data from the SoHO spacecraft, to see if this correlation has remained valid in the rising phase of solar cycle 23 and to estimate the statistical significance. We similarly evaluate anti-correlations between irradiance and cosmic ray fluxes, as observed by a number of ground-based neutron detectors.

\section{Data analysis}

\subsection{Data series employed}

We employ version 21 of the composite total solar irradiance dataset prepared and provided by PMOD/WRC, Davos. Versions 3 and 8 of this dataset are described by Fröhlich \& Lean, (1998a, 1998b), respectively. The main instruments used were HF on the Nimbus 7 satellite, ACRIM 1 on SMM, ACRIM 2 on UARS and the VIRGO instrument on SoHO. Other data considered are from the SOVA2 instrument. The main changes made since version 8 of the compilation are: the ACRIM II slip around 3 October 1995 was removed by adding $0.12 \mathrm{~W} \mathrm{~m}^{-2}$ after that date; the VIRGO data were updated to the end of May, 2001; the ACRIM II data updated and a new version from R.C Willson incorporated; the NIMBUS correction was improved (Föhlich 2000) and an improved algorithm for calculation of VIRGO data was introduced (Anklin et al. 1999; Fröhlich \& Finsterie 2001).

We compare with monthly means made from hourly averages of interplanetary magnetic field components, as observed by a variety of near-Earth satellites. These data are a continuation of the "Omnitape" dataset (Couzens \& King 1986). We also make use of cosmic ray fluxes, quantified by the count rates recorded by neutron monitors at Moscow $\left(55.47^{\circ} \mathrm{N}, 37.32^{\circ} \mathrm{E}\right.$, geomagnetic rigidity cut-off $2.46 \mathrm{GV})$, Climax $\left(39.37^{\circ} \mathrm{N},-106.18^{\circ} \mathrm{E}, 3.03 \mathrm{GV}\right)$, Hermanus $\left(-34.42^{\circ} \mathrm{N}, 19.22^{\circ} \mathrm{E}, 4.9 \mathrm{GV}\right)$, Tbilisi $\left(41.72^{\circ} \mathrm{N}\right.$, 
$\left.44.8^{\circ} \mathrm{E}, 6.91 \mathrm{GV}\right)$, Tsumeb $\left(-19.2^{\circ} \mathrm{N}, 17.6^{\circ} \mathrm{E}, 9.29 \mathrm{GV}\right)$ and Huancayo/Hawaii (13.45GV). The last pair of these stations together provide a homogeneous data sequence, the data series being continued at Haleakala, Hawaii $\left(20.72^{\circ} \mathrm{N}\right.$, $156.27^{\circ} \mathrm{E}$ ), after monitoring ceased at Huancayo, Peru $\left(-12.03^{\circ} \mathrm{N},-75.33^{\circ} \mathrm{E}\right)$ in 1993.

Figure 1 shows the variations of some of the data used. In each panel, the thin line gives monthly averages whereas the thick line gives 12-point running means of the monthly data. Figure 1a gives the variation of the cosmic ray counts observed by the neutron monitor at Climax and Fig. 1b is for Huancauyo/Hawaii. Figure 1c gives the composite variations of total solar irradiance $I_{\mathrm{TS}}$ and Fig. $1 \mathrm{~d}$ is the coronal source flux, $F_{\mathrm{S}}$, computed from near-Earth magnitude of the IMF $B_{\mathrm{sw}}$. The correlations between these parameters are discussed in Sect. 3 .

\subsection{Analysis}

We evaluate the correlation coefficient between parameters $x$ and $y$ at lag $j$ in the usual way:

$r_{j}=\frac{\left[n_{j} \sum x_{i} y_{i+j}-\sum x_{i} \sum y_{i+j}\right]}{\left[\left\{\sum x_{i}^{2}-\left(\sum x_{i}\right)^{2}\right\}\left\{\sum y_{i+j}^{2}-\left(\sum y_{i+j}\right)^{2}\right\}\right]^{1 / 2}}$

where all summations are over all i between unity and $n_{j}$, the number of pairs of data points at lag $j$. Substituting $y_{i}$ for $x_{i}$, Eq. (2) also gives the autocorrelation function of $y$, $\left[\rho_{j}\right]_{y}$. To test for significance level, $S$, equal to $100 \times(1-$ c) $\%$ where $c$ is the probability that we got the correlation by chance, we use the Students-t statistic

$t=\left|r_{j}\right|\left\{\left(N_{\mathrm{e}}-2\right) /\left(1-\left|r_{j}\right|\right)\right\}^{1 / 2}$

$N_{\mathrm{e}}$ is the effective number of independent samples, computed from the number of data points $n_{j}$, allowing for persistence (also termed "conservation") in the data (Wilks 1995):

$N_{\mathrm{e}}=n_{j}\left(1-\rho_{1}\right) /\left(1+\rho_{1}\right)$

where $\rho_{1}$ is the autocorrelation function at lag 1 , which we here take to be the mean of the autocorrelation functions at lag 1 for the two input parameters, $\left(\left[\rho_{1}\right]_{x}+\left[\rho_{1}\right]_{y}\right) / 2$.

To test the significance of a difference between two correlation coefficients we use the Fisher- $Z$ test. This involves computation of the Fisher-Z transform:

$Z(j)=0.5 \log _{e}\left\{\left(1+\left|r_{j}\right|\right) /\left(1-\left|r_{j}\right|\right)\right\}$

and $s=\{1 /(n-3)\}^{1 / 2}$. In particular, we compute $Z$ and $s$ for $r(\delta t)$ (the peak correlation that occurs at a lag $j=\delta t$ ), and for all other $j$ and $r(j)$. Hence for all $j$ we can compute

$z(j)=(Z(\delta t)-Z(j)) /\left\{s^{2}(\delta t)+s^{2}(j)\right\}^{1 / 2}$

which is normally distributed and thus the value of the significance $S(j)$ of a difference between $r(j)$ and the peak correlation $r(\delta t)$ can be computed from $z(j)$ using a normal distribution.

\subsection{Estimating the coronal source flux}

The data taken by the Ulysses spacecraft as it passed from the ecliptic plane to over the southern solar pole (Balogh et al. 1995) showed that the radial field in the heliosphere was approximately independent of latitude, once allowance has been made for the expected $r_{\mathrm{s}}^{2}$ variation with heliocentric distance, $r_{\mathrm{s}}$. Lockwood et al. (1999b) have shown that this was also true for the pole-to-pole fast-latitude perihelion pass. The result has been explained by Suess \& Smith (1996) and Suess et al. (1996) in terms of the pressure transverse to the flow in the expanding solar wind at $r_{\mathrm{s}}$ between about 2.5 and $10 R_{\mathrm{s}}\left(1 R_{\mathrm{s}}\right.$ is a mean solar radius) where the plasma beta is very low.

The coronal source flux has also been estimated from measurements of the line-of-sight component of the photospheric field (at $r=1 R_{\mathrm{s}}$ ). In deriving this line-of-sight component of the field from magnetograph data for the central solar meridian, a latitude-dependent "saturation" correction factor must be applied (Wang \& Sheeley 1995). The radial component is then computed by dividing by a cosine factor (so there is no information from over the solar poles). The open flux is then estimated using a method such as the potential field source surface (PFSS) procedure (Schatten et al. 1969), in which the coronal field is assumed to be current-free between the photospheric surface and the coronal source surface, where the field is assumed to be radial. With an improved latitude-dependent saturation correction factor, Wang \& Sheeley (1995) were able to match to the radial field seen at Earth during solar cycles 20 and 21, again using the assumption that $B_{\mathrm{r}}$ is independent of latitude in the heliosphere, as found from the Ulysses observations.

Because of this result, the radial field seen at Earth $B_{\mathrm{rE}}$ can be used to compute the total flux threading a heliocentric sphere of radius $R_{1}=1 \mathrm{AU}$. Neglecting the small flux threading the heliospheric current sheet between $r_{\mathrm{s}}=2.5 R_{\mathrm{s}}$ and $r_{\mathrm{s}}=R_{1}$, this equals the total open flux threading the coronal source surface which is an approximately spherical at $r \approx 2.5 R_{\mathrm{s}}$ and this can be computed from:

$F_{\mathrm{S}}=B_{\mathrm{rE}} \cdot 4 \pi R_{1}^{2} / 2$.

The factor 2 arises because half the flux through this surface is outward (away from the Sun) and half is inward.

Parker spiral theory predicts the heliospheric field components in heliocentric polar $\left(r_{\mathrm{s}}, \phi, \psi\right)$ coordinates will be:

$$
\begin{aligned}
B_{\mathrm{sw}} & =\left\{B_{\mathrm{r}}^{2}+B_{\phi}^{2}+B_{\psi}^{2}\right\}^{1 / 2} \\
& =B_{\mathrm{r}}\left\{1+\tan ^{2} \gamma\right\}^{1 / 2} \\
& =B_{\mathrm{o}}\left(R_{\mathrm{o}} / r_{\mathrm{s}}\right)^{2}\left\{1+\left(\omega r_{\mathrm{s}} \cos \psi / \mathrm{v}_{\mathrm{sw}}\right)^{2}\right\}^{1 / 2}
\end{aligned}
$$

Stamper et al. (1999) and Gazis (1996) have shown that this theory correctly predicts $B_{\psi}=0$ and the garden hose angle $\gamma$ in annual means. In addition, the value of $\gamma$ is almost constant in annual mean data from near Earth. Thus the coronal source flux is also given by:

$F_{\mathrm{S}}=B_{\mathrm{sw}} \cdot 2 \pi R_{1}^{2} /\left\{1+\tan ^{2} \gamma\right\}^{1 / 2}$. 

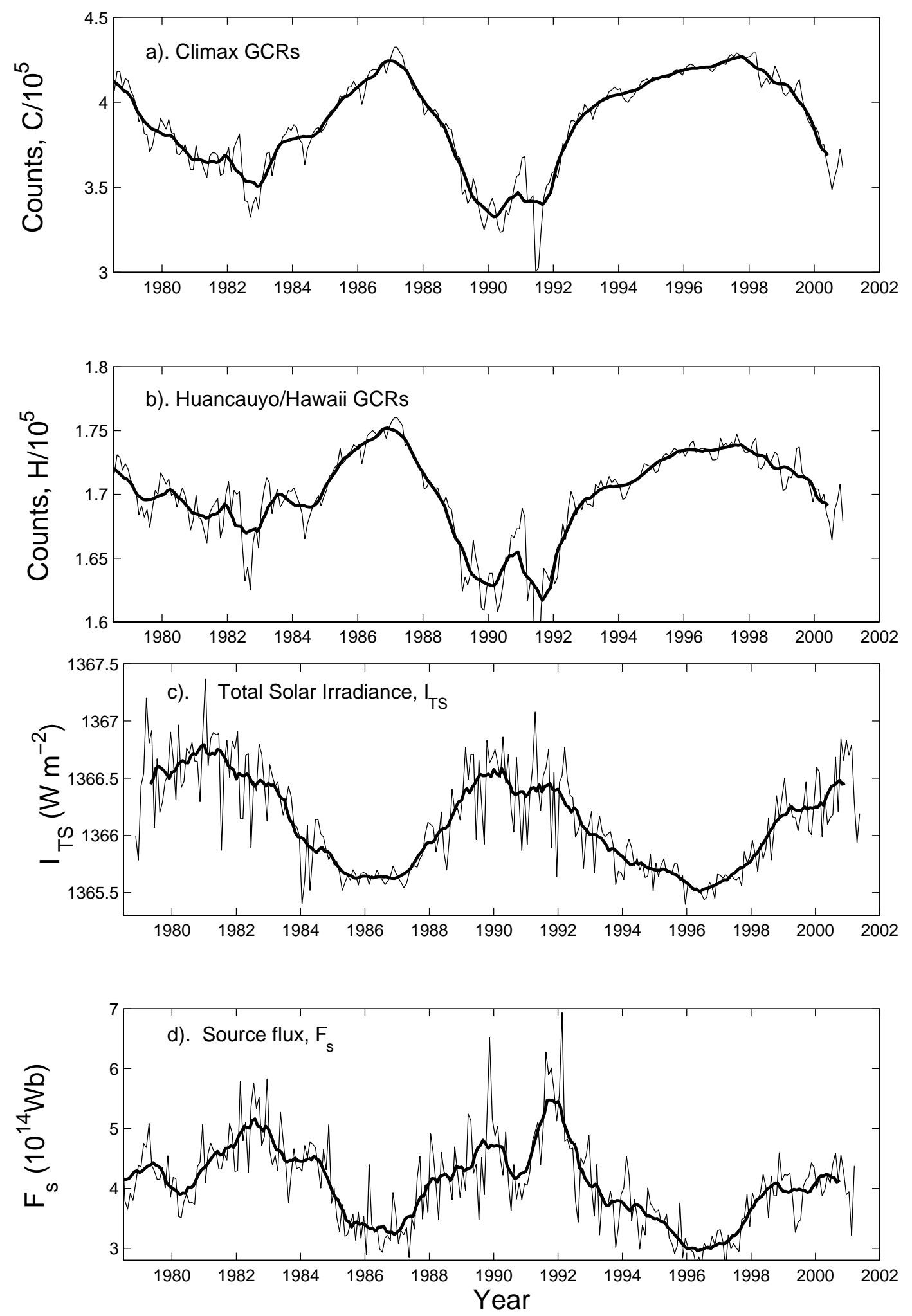

Fig. 1. Variations of the cosmic ray flux observed by neutron monitors at a) Climax ( $>3$ GV) and b) Huancauyo/Hawaii $(>13 \mathrm{GV})$; of c) the total solar irradiance, $I_{\mathrm{TS}}$; and $\mathbf{d}$ ) of the coronal source flux, $F_{\mathrm{S}}$, computed from near-Earth magnitude of the IMF $B_{\mathrm{sw}}$. In each case the thin line shows monthly averages whereas the thick solid line is the 12-month running mean.

We use both Eqs. (7) and (9), the latter with the overall average of $\gamma$, to determine $F_{\mathrm{S}}$. The disadvantage of (7) is that there is more short-term variability in $B_{\mathrm{r}}$ than in $B_{\mathrm{sw}}$, because on shorter timescales the field is subject to deflection in direction away from the Parker spiral direction, for example as it is draped over coronal mass ejections and warped by co-rotation interaction regions and the corrugated current sheet. These deflections change $B_{\mathrm{r}}$ 


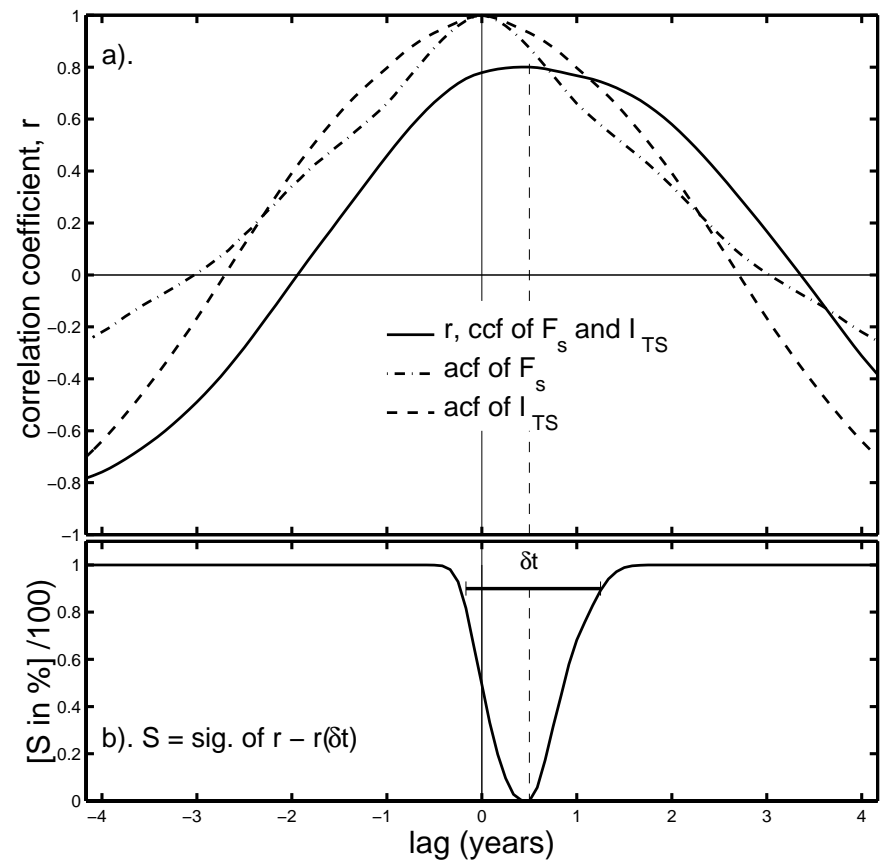

Fig. 2. a) Correlogram of the total solar irradiance $I_{\mathrm{TS}}$ and the coronal source flux $F_{\mathrm{S}}$ computed from near-Earth magnitude of the IMF $B_{\mathrm{sw}}$. A positive lag is defined as the $F_{\mathrm{S}}$ sequence being lagged. The solid line is the cross-correlation function (ccf, $r$ ), the dashed line the autocorrelation function (acf) of $I_{\mathrm{TS}}$, and the dot-dash line the acf of $F_{\mathrm{S}}$. The vertical dashed line gives the lag $\delta t=6$ months at which $|r|$ is a maximum. b) The significance $S$ of the difference between $r(t)$ and $r(\delta t)$. The horizontal bar gives the uncertainty in $\delta t$ set by $S=90 \%$. These plots are for 12-month running means of monthly data.

but do not influence the total open solar flux: therefore in many ways it is better to use Eq. (9), with the average value of $\gamma$, to compute $F_{\mathrm{S}}$. In this paper we use both $(7)$ and (9) and derive similar answers.

\section{Results}

\subsection{The correlation between IMF and irradiance}

Figure 2a shows the correlogram for the total solar irradiance $I_{\mathrm{TS}}$ and the coronal source flux $F_{\mathrm{S}}$, computed from near-Earth magnitude of the IMF, $B_{\mathrm{sw}}$, using Eq. (9). A positive lag is defined as the $F_{\mathrm{S}}$ sequence being lagged (i.e. the $F_{\mathrm{S}}$ variation occurring before the corresponding $I_{\mathrm{TS}}$ variation). The solid line is the cross-correlation function (ccf, $r$ ), the dashed line the autocorrelation function (acf, $\rho$ ) of $I_{\mathrm{TS}}$ and the dot-dash line the acf of $F_{\mathrm{S}}$. The vertical dashed line gives the lag $\delta t$ at which the absolute value of the ccf $|r|$ is a maximum. This plot is for 12-month running means of monthly data. Because it is dealing with yearly averages, the peak correlation of $r(\delta t)=0.865$ is similar to that reported by Lockwood \& Stamper (1999), but not exactly the same because these authors used an $F_{\mathrm{S}}$ estimate derived from the aa geomagnetic index. This high correlation means that $r^{2}(\delta t)=0.64$ of the variation in $I_{\mathrm{TS}}$ can be associated with $F_{\mathrm{S}}$. This occurs at a

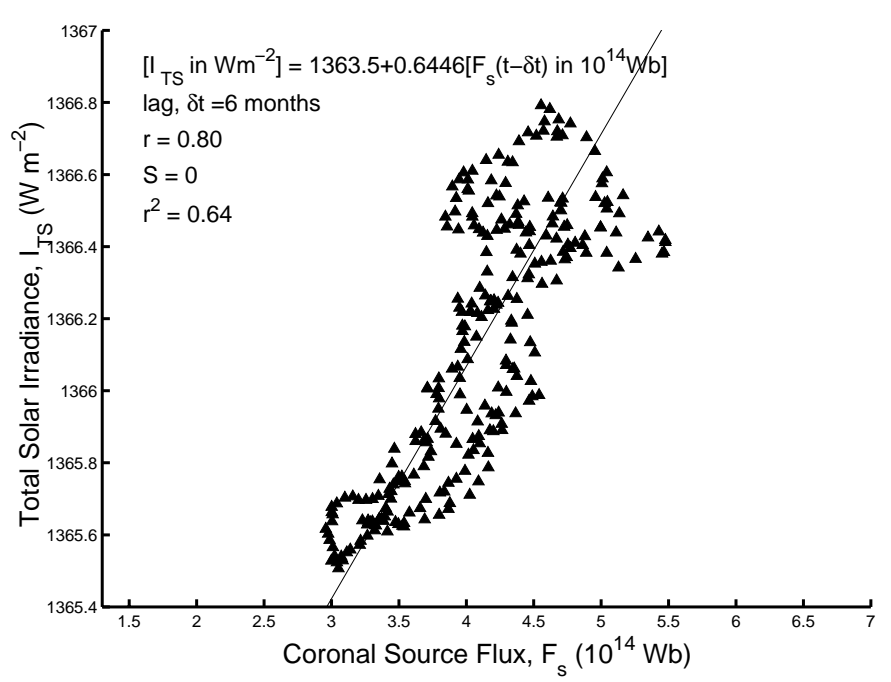

Fig. 3. Scatter plot of the total solar irradiance $I_{\mathrm{TS}}(t)$ and the lagged coronal source flux $F_{\mathrm{S}}(t-\delta t)$ (so the $F_{\mathrm{S}}$ data series has been lagged in time by $\delta t=+6$ months). $F_{\mathrm{S}}$ has been computed from near-Earth magnitude of the IMF $B_{\mathrm{sw}}$ using Eq. (9). The line gives the best-fit linear regression fit. This plot is for 12-month running means of monthly data.

lag $\delta t=6$ months. Figure $2 \mathrm{~b}$ analyses the uncertainty in this lag by showing the significance $S(j)$ of the difference between $r(j)$ and $r(\delta t)$, calculated using the Fischer-Z test described in Sect. 2.2. It can be seen that the minimum in $S(j)$ around $S(\delta t)=0$ is well defined and that the upper and lower uncertainty limits (at the $90 \%$ level, marked by the horizontal bar) are -2 and 15 months.

Figure 3 gives the scatter plot between $F_{\mathrm{S}}$ and the irradiance $I_{\mathrm{TS}}$ for the otimum lag $\delta t=6$ months . Although $r(\delta t)$ for this smoothed data is very high, the lag-one autocorrelation coefficients $\rho_{1}$ are high for both $F_{\mathrm{S}}$ and $I_{\mathrm{TS}}$, as can be seen in Fig. 2a. As a result, Eq. (4) yields $N_{\mathrm{e}}<2$ and thus the $t$ value becomes complex and we cannot ascribe any significance to this correlation. The line in Fig. 3 is the best-fit linear regression, which has been used to scale the $F_{\mathrm{S}}$ data (lagged by $\delta t$ ) onto the $I_{\mathrm{TS}}$ axis in Fig. 4 so that the temporal variations can be compared. All the best-fit coefficients are listed in Tables 1 and 2 .

Figures 2-4 show that although an excellent correlation is obtained in these 12 -month average values, the smoothing has introduced persistence in the data to such an extent that the correlation has no significance. In order to get a significant result $\left(N_{\mathrm{e}}>2\right)$, this correlation would need to be maintained in a similarly-smoothed data series covering at least 48 years.

Without the smoothing, we obtain a lower, but statistically very significant correlation. This is demonstrated by Figs. 5-7 which are the same as Figs. 2-4, but for unsmoothed monthly data. Table 1 shows that the peak correlation coefficient $r(\delta t)=0.62$ (meaning that only $r^{2}(\delta t)=0.39$ of the variation in $I_{\mathrm{TS}}$ can be associated with that in $F_{\mathrm{S}}$ ). However this correlation is significant at the $99.999 \%$ level. Again, all coefficients of this fit are given in Tables 1 and 2 . 


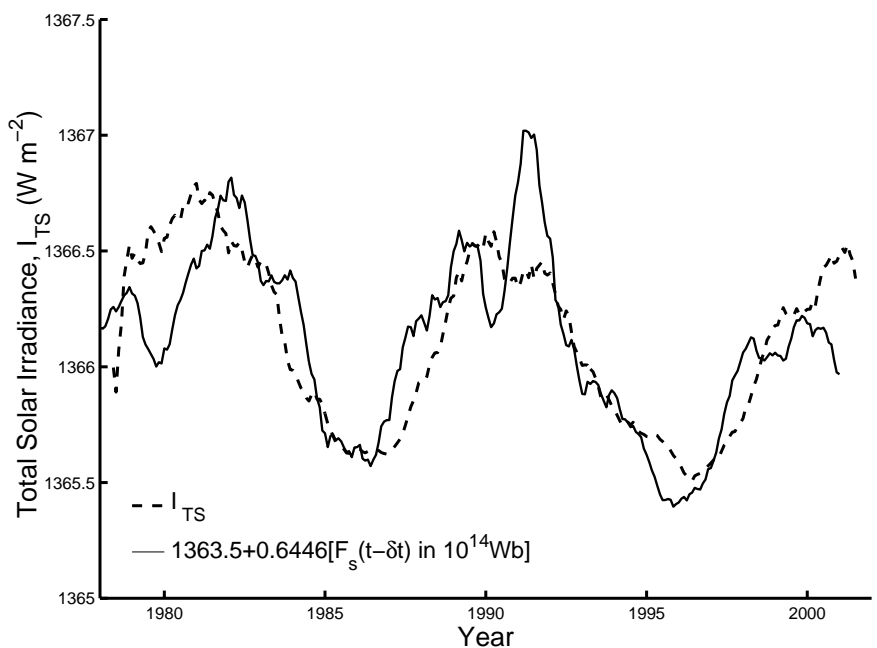

Fig. 4. Temporal variations of the total solar irradiance observed $I_{\mathrm{TS}}$ (thick dashed line) and scaled (thin solid line) using the linear regression fit in Fig. 3 from the coronal source flux $F_{\mathrm{S}}$, lagged in time by $\delta t=+6$ months. $F_{\mathrm{S}}$ has been computed from near-Earth magnitude of the IMF $B_{\mathrm{sw}}$. This plot is for 12-month running means of monthly data.

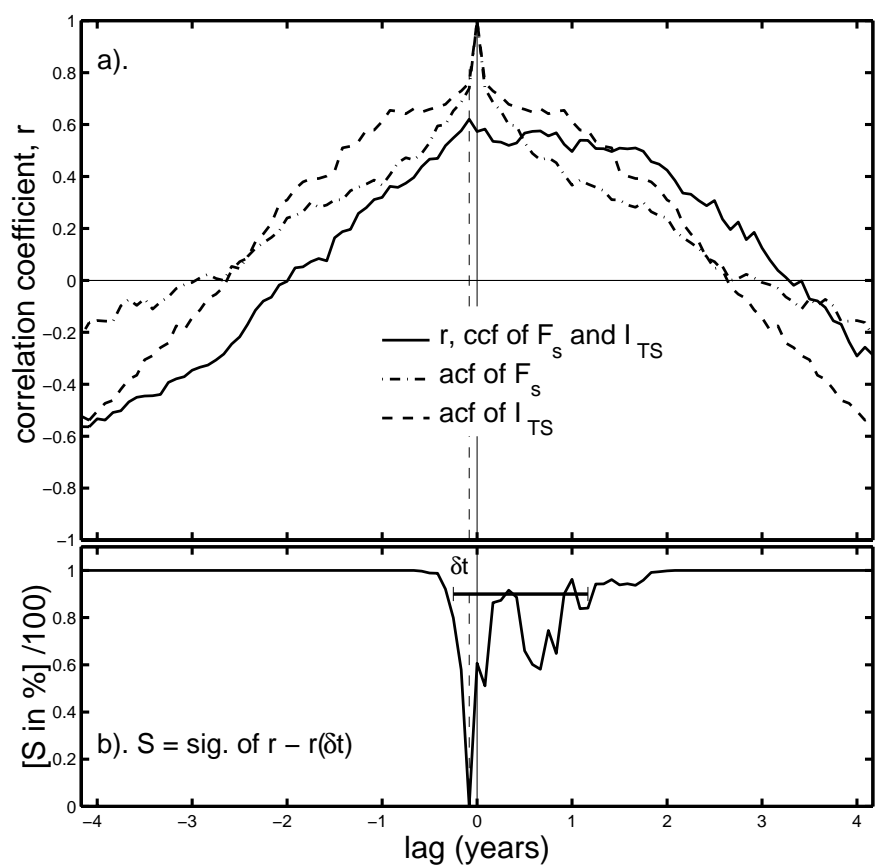

Fig. 5. Same as Fig. 2 for monthly mean data. The peak correlation is at $\delta t=-1$ month.

Tables 1 and 2 also give the results if Eq. (7) and the observed radial field are used to compute the open solar flux (denoted by $F_{S}^{*}$ ). As expected, the additional variability in $B_{\mathrm{r}}$, introduced by field line draping and warping over heliospheric structure, has reduced the correlation and its significance somewhat.

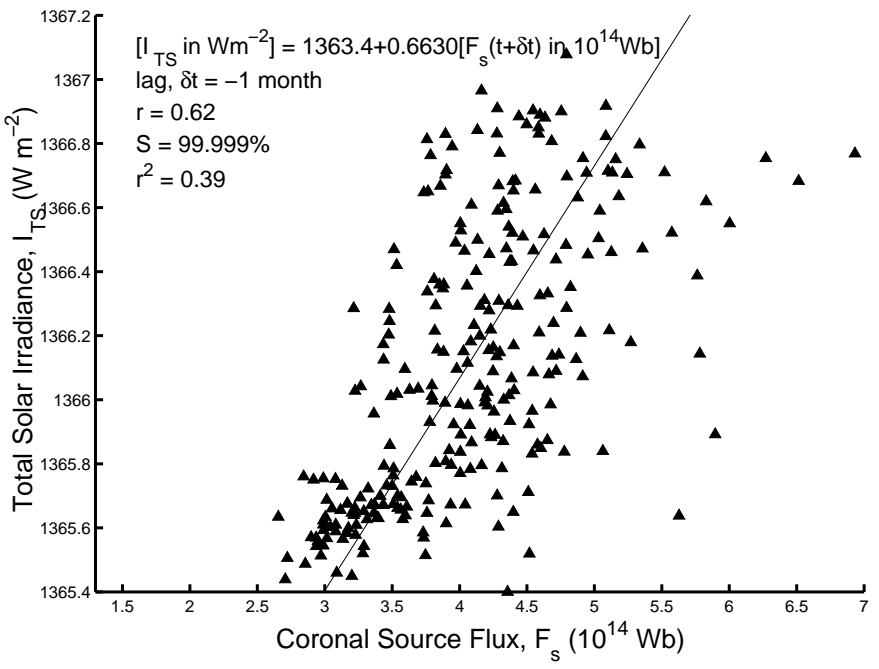

Fig. 6. Same as Fig. 3 for monthly mean data. The lag giving peak collaboration is $\delta t=-1$ month.

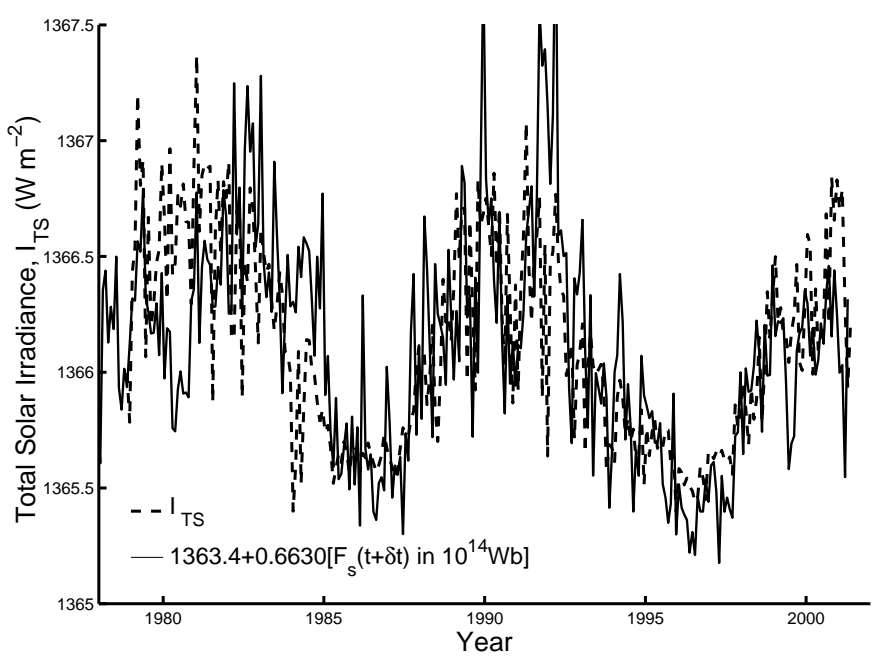

Fig. 7. Same as Fig. 4 for monthly mean data. The lag giving peak collaboration is $\delta t=-1$ month.

\subsection{The correlation between cosmic rays and irradiance}

Lockwood (2001) has shown that of order $80 \%$ of the overall variation in annual means of fluxes of galactic cosmic rays impacting Earth's atmosphere is explained by the variation in the strength of the heliospheric field which contributes to the diffusive barrier that shields the inner heliosphere (Moraal 1993; Potgieter 1995). There are other mechanisms that shield cosmic rays (Jokipii 1991). In particular, polarity-dependent curvature and gradient drifts are a factor at solar minimum (Ahluwalai 1997; Ahluwalia \& Wilson 1996; Usoskin et al. 1998; Cane et al. 1999). On the other hand, because they reflect the threedimensional shielding of the heliosphere, cosmic rays are not subject to the local perturbations that influence the near-Earth heliospheric field measurements.

Figures 8-11 investigate the anti-correlation between $I_{\mathrm{TS}}$ and the Moscow neutron monitor counts, $M$, in the 
Table 1. Parameters quantifying the linear correlations between total solar irradiance and various indicators of the open solar flux.

\begin{tabular}{|c|c|c|c|c|c|c|c|}
\hline & $\begin{array}{l}\text { Dates } \\
\text { (inclusive) }\end{array}$ & $\begin{array}{l}\text { Correlation } \\
\text { coefficient, } \\
r\end{array}$ & $\begin{array}{l}\text { Fraction of } \\
\text { variation } \\
\text { explained, } \\
r^{2}\end{array}$ & $\begin{array}{l}\text { Number of } \\
\text { pairs of } \\
\text { data points, } \\
N\end{array}$ & $\begin{array}{l}\text { Average } \\
\mathrm{ACF} \text { at lag } \\
1, \rho_{1}\end{array}$ & $\begin{array}{l}\text { Effective } \\
\text { number of } \\
\text { samples, } N_{\mathrm{e}}\end{array}$ & $\begin{array}{l}\text { Significance } \\
S(\%)\end{array}$ \\
\hline$\left\langle F_{\mathrm{S}}\right\rangle$ and $\left\langle I_{\mathrm{TS}}\right\rangle$ & $\begin{array}{l}\text { Jun. } 1979- \\
\text { Nov. } 2000\end{array}$ & 0.800 & 0.641 & 272 & 0.994 & 0.85 & 0 \\
\hline$\left\langle F_{\mathrm{S}}^{*}\right\rangle$ and $\left\langle I_{\mathrm{TS}}\right\rangle$ & $\begin{array}{l}\text { Jun. } 1979- \\
\text { Nov. } 2000\end{array}$ & 0.666 & 0.444 & 261 & 0.993 & 0.91 & 0 \\
\hline$F_{\mathrm{S}}$ and $I_{\mathrm{TS}}$ & $\begin{array}{l}\text { Oct. } 1978 \text { - } \\
\text { Mar. } 2001\end{array}$ & 0.621 & 0.385 & 270 & 0.748 & 38.8 & 99.999 \\
\hline$F_{\mathrm{S}}^{*}$ and $I_{\mathrm{TS}}$ & $\begin{array}{l}\text { Oct. } 1978 \text { - } \\
\text { Mar. } 2001\end{array}$ & 0.489 & 0.240 & 255 & 0.725 & 40.5 & 99.94 \\
\hline$C$ and $I_{\mathrm{TS}}$ & $\begin{array}{l}\text { Oct. } 1979- \\
\text { Nov. } 2000\end{array}$ & -0.716 & 0.513 & 262 & 0.970 & 3.9 & 85.5 \\
\hline$H_{\mathrm{e}}$ and $I_{\mathrm{TS}}$ & $\begin{array}{l}\text { Oct. } 1979 \text { - } \\
\text { Nov. } 1999\end{array}$ & -0.736 & 0.542 & 239 & 0.961 & 4.8 & 91.6 \\
\hline$T_{\mathrm{b}}$ and $I_{\mathrm{TS}}$ & $\begin{array}{l}\text { Oct. } 1979- \\
\text { Sep. } 1997\end{array}$ & -0.805 & 0.649 & 230 & 0.939 & 5.5 & 95.8 \\
\hline$T_{\mathrm{s}}$ and $I_{\mathrm{TS}}$ & $\begin{array}{l}\text { Oct. } 1979- \\
\text { Jan. } 1999\end{array}$ & -0.785 & 0.617 & 230 & 0.939 & 7.3 & 98.3 \\
\hline$H$ and $I_{\mathrm{TS}}$ & $\begin{array}{l}\text { Oct. } 1979- \\
\text { Oct. } 2000\end{array}$ & -0.832 & 0.692 & 252 & 0.971 & 3.7 & 90.3 \\
\hline$M$ and $I_{\mathrm{TS}}$ & $\begin{array}{l}\text { Oct. } 1979- \\
\text { Nov. } 2000\end{array}$ & -0.713 & 0.508 & 262 & 0.966 & 4.6 & 90.3 \\
\hline$\langle M\rangle$ and $\left\langle I_{\mathrm{TS}}\right\rangle$ & $\begin{array}{l}\text { Mar. } 1982- \\
\text { May } 2000\end{array}$ & -0.929 & 0.863 & 219 & 0.997 & 0.31 & 0 \\
\hline
\end{tabular}

* Using $B_{\mathrm{r}}$ and Eq. (7).

Table 2. Coefficients from linear regressions $\left[I_{\mathrm{TS}}(t)\right.$ in $\left.\mathrm{Wm}^{-2}\right]=s A(t-\delta t)+c$ associated with the correlations discussed in Table 1.

\begin{tabular}{|c|c|c|c|c|c|c|}
\hline $\mathrm{A}$ & $\begin{array}{l}\text { Dates } \\
\text { (inclusive) }\end{array}$ & Slope, $s$ & Intercept, $c$ & $\begin{array}{l}\text { Lag, } \delta t \\
\text { (months) }\end{array}$ & $\begin{array}{l}\min \delta t \\
\text { (months) }\end{array}$ & $\begin{array}{l}\max \delta t \\
\text { (months) }\end{array}$ \\
\hline$\left\langle F_{\mathrm{S}}\right\rangle\left(\right.$ in $\left.10^{14} \mathrm{~Wb}\right)$ & $\begin{array}{l}\text { Jun. } 1979- \\
\text { Nov. } 2000\end{array}$ & 0.645 & 1363.5 & 6 & -2 & 15 \\
\hline$\left\langle F_{\mathrm{S}}^{*}\right\rangle\left(\right.$ in $\left.10^{14} \mathrm{~Wb}\right)$ & $\begin{array}{l}\text { Jun. } 1979- \\
\text { Nov. } 2000\end{array}$ & 0.486 & 1364.1 & 14 & 7 & 26 \\
\hline$F_{\mathrm{S}}\left(\right.$ in $\left.10^{14} \mathrm{~Wb}\right)$ & $\begin{array}{l}\text { Oct. } 1978- \\
\text { Mar. } 2001\end{array}$ & 0.663 & 1363.4 & -1 & -3 & 14 \\
\hline$F_{\mathrm{S}}^{*}\left(\right.$ in $\left.10^{14} \mathrm{~Wb}\right)$ & $\begin{array}{l}\text { Oct. } 1978- \\
\text { Mar. } 2001\end{array}$ & 0.501 & 1364.0 & 14 & -2 & 29 \\
\hline $\begin{array}{l}C \text { (Climax count } \\
\text { rate) }\end{array}$ & $\begin{array}{l}\text { Oct. } 1979- \\
\text { Nov. } 2000\end{array}$ & $-1.604 \times 10^{-4}$ & 1372.3 & 3 & -2 & 17 \\
\hline $\begin{array}{l}H_{\mathrm{e}} \text { (Hermanus } \\
\text { Count Rate) }\end{array}$ & $\begin{array}{l}\text { Oct. } 1979- \\
\text { Nov. } 1999\end{array}$ & -0.0024 & 1376.1 & 3 & -2 & 13 \\
\hline $\begin{array}{l}T_{\mathrm{b}} \text { (Tbilisi Count } \\
\text { Rate) }\end{array}$ & $\begin{array}{l}\text { Oct. } 1979- \\
\text { Sep. } 1997\end{array}$ & -0.0013 & 1377.5 & 2 & -3 & 18 \\
\hline $\begin{array}{l}T_{\mathrm{s}} \text { (Tsumeb Count } \\
\text { Rate) }\end{array}$ & $\begin{array}{l}\text { Oct. } 1979- \\
\text { Jan. } 1999\end{array}$ & -0.0014 & 1382.5 & 3 & -2 & 18 \\
\hline $\begin{array}{l}H \text { (Huancayo/ } \\
\text { Hawaii count rate) }\end{array}$ & $\begin{array}{l}\text { Oct. } 1979- \\
\text { Oct. } 2000\end{array}$ & $-1.338 \times 10^{-4}$ & 1.388 .9 & -1 & -3 & 17 \\
\hline $\begin{array}{l}M \text { (Moscow Count } \\
\text { Rate) }\end{array}$ & $\begin{array}{l}\text { Oct. } 1979- \\
\text { Nov. } 2000\end{array}$ & $-8.986 \times 10^{-6}$ & 1373.9 & 3 & -2 & 17 \\
\hline $\begin{array}{l}\langle M\rangle \text { (Moscow } \\
\text { Count Rate) }\end{array}$ & $\begin{array}{l}\text { Mar. } 1982- \\
\text { May } 2000\end{array}$ & $-6.485 \times 10^{-6}$ & 1371.7 & 4 & 1 & 8 \\
\hline
\end{tabular}

* Using $B_{\mathrm{r}}$ and Eq. (7). 


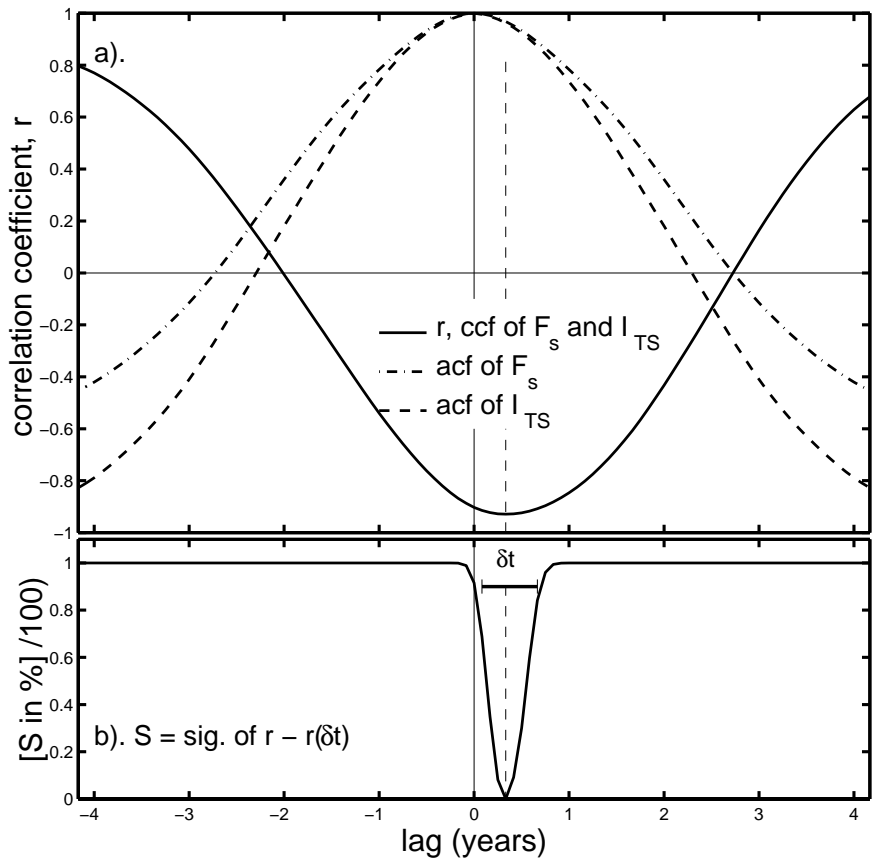

Fig. 8. Same as Fig. 2 for total solar irradiance $I_{\mathrm{TS}}$ and cosmic ray counts $M$ detected by the neutron monitor at Moscow. A positive lag is defined as the $M$ data sequence being lagged. These plots are for 12-point running means of monthly averages for $1982-2001$.

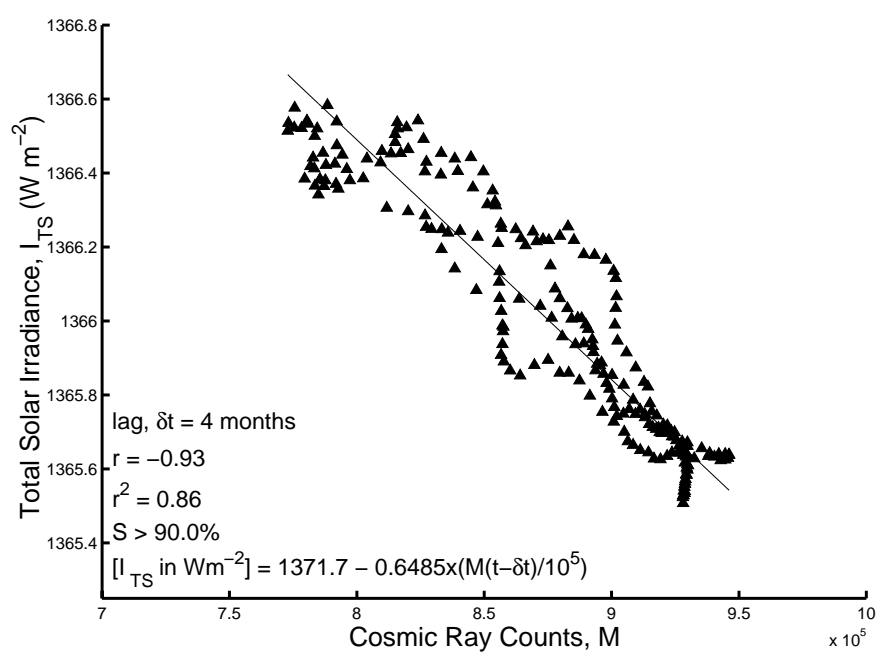

Fig. 9. Same as Fig. 3, for 12-point running means of monthly averages of total solar irradiance $I_{\mathrm{TS}}$ and the lagged (by $\delta t=$ 4 months) cosmic ray counts $M$, as detected by the neutron monitor at Moscow. The data and regression fit shown are for $I_{\text {TS }}$ data after 1 January 1982 only: details of the corresponding fit for all data are given in Tables 1 and 2 .

same format as Figs. 2-5. The results are also summarised in Tables 1 and 2. The correlation has been done in two ways. Figure 11 is for monthly means, and all the available $I_{\mathrm{TS}}$ data were used. As noted by Lockwood \& Stamper (1999), the first 2 years of $I_{\mathrm{TS}}$ measurements do not correlate as well with $F_{\mathrm{S}}$ and we here stress this by repeating the analysis for 12-month running means of $I_{\mathrm{TS}}$ data after March 1982 only. In Figs. 8 and 9, only data from after

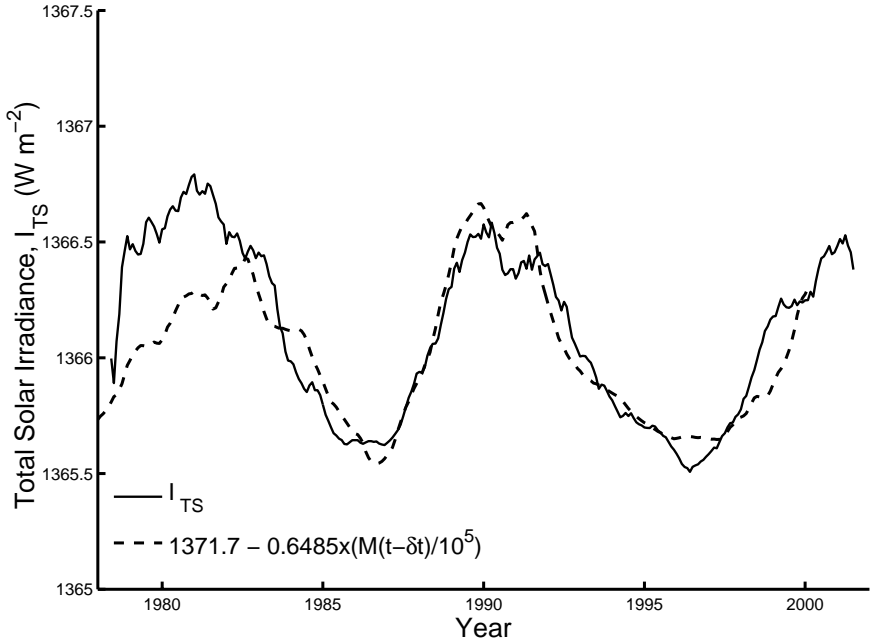

Fig. 10. Same as figure 4, for 12-month running means of monthly averages of total solar irradiance $I_{\mathrm{TS}}$ and the lagged (by $\delta t=4$ months) cosmic ray counts $M$ detected by the neutron monitor at Moscow, scaled using the regression line shown in Fig. 9.

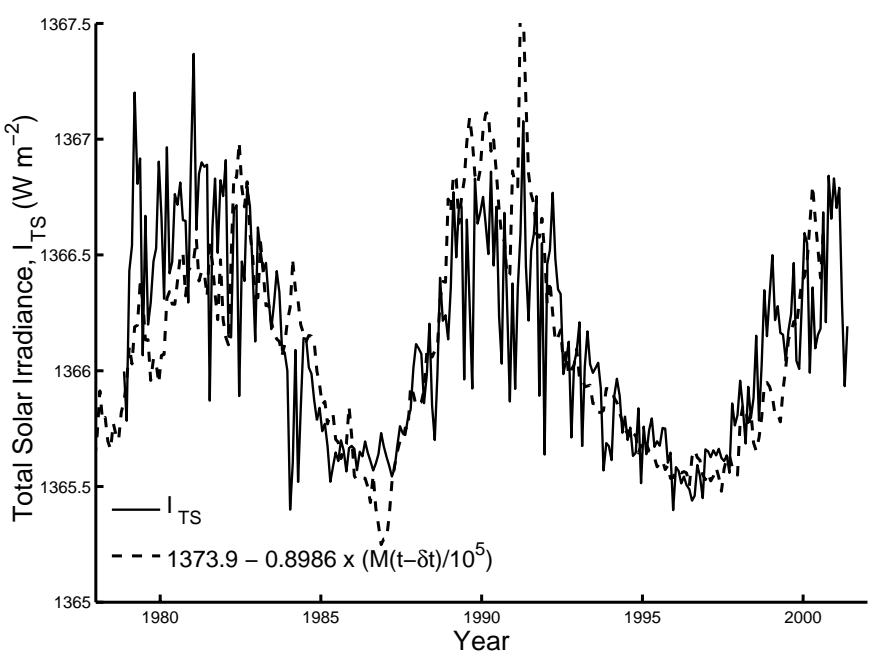

Fig. 11. Same as Fig. 10, but for monthly data and using a regression fit of all monthly means. The best-fit lag is $\delta t=$ 3 months.

this date are included. The best fit shown in Fig. 9 is used to scale the whole of the data sequence in Fig. 10. It can be seen that the agreement is very strong after 1982 $\left(r(\delta t)=0.93, r^{2}(\delta t)=0.86\right)$ but poorer before then.

Inspection of Figs. 10 and 11 indicates that the offset between the observed $I_{\mathrm{TS}}$ and the scaled $M$ variation appears to grow as we go back in time from about the end of 1983 to mid 1980. The same behaviour can be seen in Fig. 7. This could be interpreted as revealing that the early progressive degradation of the instruments has been underestimated in the composite irradiance data series. Full calibration of the degradation of instruments is achieved by using two identical radiometers and monitoring the ratio of the responses of the fully operational instrument and the calibration instrument (which is only rarely exposed). For the early data, ACRIM-1/SSM had a 
back up instrument that was only used very rarely, but the HF/Nimbus7 instrument did not and the predicted degradation for the latter relies mainly on theoretical expectations and comparison with the similar PM06V radiometer which is part of the VIRGO experiment on SoHO. Figure 12 plots the variation of the deviation of the fit from the observations for monthly means (thin line), along with that for 12-month running means of these monthly data (thick line). The dashed lines mark plus and minus one r.m.s. value of the deviation in monthly values and the early data (before January 1982) are the only ones that are consistently outside these. Comparison with a JPL rocket experiment in 1980 (compared to other similar comparisons at later times) lends some support for the idea that the composite values might be up to about $0.25 \mathrm{~W} \mathrm{~m}^{-2}$ too high at this time (see Fröhlich \& Lean 1998a, 1998b). Such a change would also improve the agreement with $F_{\mathrm{S}}$ inferred from IMF data (see Fig. 7), but not eliminate the discrepancy entirely.

However, Fig. 12 also shows that the monthly data at the very start of the irradiance data series (for 1978) do agree well with the best fit and this argues against the idea that the progressive instrument degradation has been underestimated. There is also no obvious observational reason as to why this should be the case. Thus it seems likely that this discrepancy reveals a long-term trend in one or both of the parameters and so highlights the limitations of the correlation discussed here, rather than reflecting an observation and/or inter-calibration problem.

The behaviour seen in data from the Moscow neutron monitor is very similar indeed to that in data from other neutron monitors, as shown by the various correlations given in Tables 1 and 2. No consistent trend with the geomagnetic rigidity cut off is found.

\section{Discussion and conclusions}

We find that the correlation between open solar flux and solar irradiance reported by Lockwood \& Stamper (1999) has passed the test provided by the addition of 5 years' more data from the rising phase of cycle 23. The lower correlation found in monthly data $(r=0.62)$ is highly significant statistically $(99.999 \%)$, whereas the higher correlation for annual means $(r=0.87)$ will require it to be maintained in more than twice as much data before it could be considered significant. The correlation is expected to be poorer on shorter scales because individual photospheric magnetic features, such as for example a sunspot group, can grow and fade on timescales equivalent to, or shorter than, the 1-month averaging intervals used here and so produce rapid variations in $I_{\mathrm{TS}}$. On the other hand, open flux variations change on longer time scales associated with open flux emergence and decay (Solanki et al. 2000). Significant anticorrelations are found with cosmic rays for the full range of geomagnetic rigidity cut-offs $(2.4-13 \mathrm{GV})$. All data imply that the irradiance lags behind the open solar flux. Combining the values from all independent data on cosmic rays and the IMF

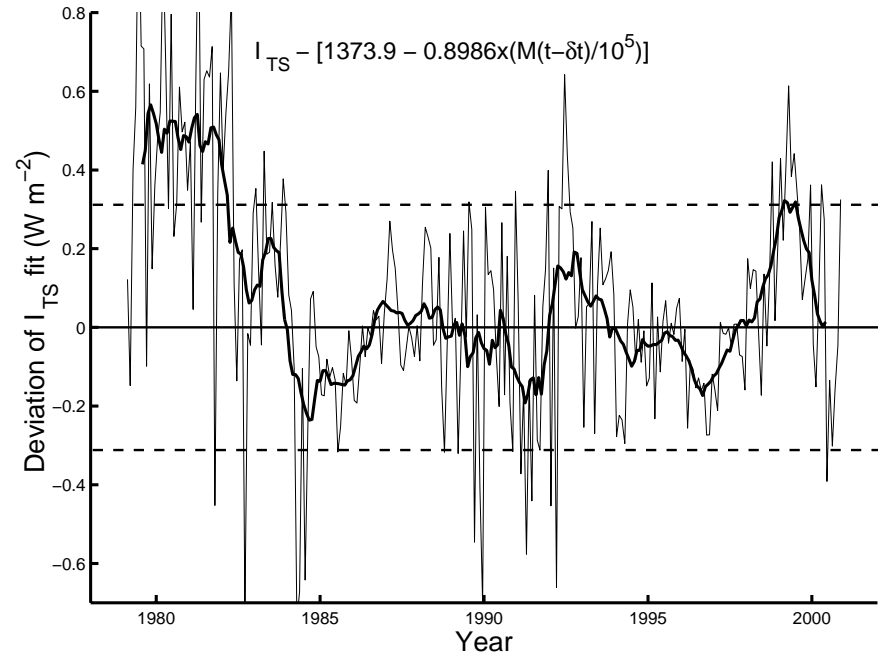

Fig. 12. The deviation of the best fit total irradiance from the value $I_{\mathrm{TS}}$ (from Figs. 10 and 11). The thin line is for monthly means; the thick solid line is for 1-year running means. The horizontal dashed lines are plus and minus one RMS value of the deviation for monthly data and for the whole period.

(by assuming bi-normal distributions about $\delta t$ ) gives an optimum lag of 2.8 months (and within the uncertainty range $0.8-8.0$ months at the $90 \%$ level).

We do not know what physical mechanism could be active to generate the correlations discussed in this paper. It could have little significance beyond the fact that there is a solar cycle variation in both $F_{\mathrm{S}}$ and $I_{\mathrm{TS}}$. The more detailed similarities between the two, however, imply that the open magnetic flux in some way quantifies both the spectrum of flux tube dimensions and the total flux of magnetic flux tubes threading the photosphere. Solanki et al. (2000) obtained a good match to both the open flux variation derived by Lockwood et al. (1999a) and to the cosmic ray flux variation inferred from the ${ }^{10}$ Be cosmogenic isotope (Beer et al. 2000; Lockwood 2001; McCracken \& McDonald 2001) using a simple model of open flux emergence in active regions and its subsequent decay. Recently, Solanki et al. (2001) have extended this modelling to include flux emergence in ephemeral regions and to estimate the total photospheric flux. Interestingly, the variation in the total photospheric flux that they derive is very similar indeed in form to the open flux variation which again matches the open flux variation found by Lockwood et al. (1999a). This work therefore implies that the open flux, despite being only a few percent of the total photospheric flux, may nevertheless be a valuable proxy for it.

As well as its potential value in irradiance variation reconstruction, one important aspect of this correlation, if confirmed and understood, would lie in the fact that it would provide one link between records of the abundances of cosmogenic isotopes and solar climate forcing. The ${ }^{10} \mathrm{Be}$ isotope is produced in the atmosphere as a spallation product when cosmic rays impact upon oxygen and nitrogen in the atmosphere. The precipitation into the ice sheets means that the abundances found there 
are convolved with a climate influence that could be a factor in the long-term variation (Beer 2000; Lockwood 2001). The ${ }^{14} \mathrm{C}$ isotope is, on the other hand, absorbed into tree rings directly in the gaseous state. However, there are different complications in this case because the oceans and the biosphere act as large reservoirs which disconnect abundances and production rates (Stuiver \& Quay 1980). Despite these differences, modelling of the effect of ${ }^{14} \mathrm{C}$ reservoirs implies that the production rates of ${ }^{14} \mathrm{C}$ and ${ }^{10} \mathrm{Be}$ are similar (Bard et al. 1997; Beer 2000). This suggests that similarities between the cosmogenic isotope record and climate records indicate a real link to climate forcing.

Acknowledgements. The author is grateful to PMOD/WRC, Davos, Switzerland for version 21 of the composite total solar irradiance dataset, which includes unpublished data from the VIRGO Experiment on the cooperative ESA/NASA Mission SoHO. He also grateful to the World Data Center system for collecting, archiving, and distributing the cosmic ray and interplanetary data and to the many scientists who contributed these data to the WDC network. He also thanks a number of scientists for discussions and pre-prints concerning their recent work: in particular, S. Solanki, C. Fröhlich, Y.-M. Wang, and J. Lean. This work was supported by the U.K. Particle Physics and Astronomy Research Council.

\section{References}

Ahluwalia, H. S. 1997, J. Geophys. Res., 102, 24, 229

Ahluwalia, H. S., \& Wilson, M. D. 1996, J. Geophys. Res., 101, 4879

Anklin, M., Fröhlich, C., Finsterle, W., Crommelynck, D. A., \& Dewitte, S. 1999, Metrologia 35, 685

Balogh, A., Smith, E. J., Tsurutani, B. T., et al. 1995, Science, 268, 1007

Bard, E., Raisbeck, G. M., Yiou, F., \& Jouzel, J. 1997, Earth and Planet. Sci. Lett., 150, 453

Beer, J. 2000, Space Sci. Rev., 94(1/2), 53

Cane, H. V., Wibberenz, G., Richardson, I. G., \& von Rosenvinge, T. T. 1999, Geophys. Res. Lett., 26, 565

Chapman, G. A., Cookson, A. M., \& Dobias, J. J. 1997, Astrophys. J., 842, 541

Couzens, D. A., \& King, J. H. 1986, Interplanetary Medium Data Book - Supplement 3, National Space Science Data Center (Goddard Space Flight Center, Greenbelt, Maryland, USA)

Fligge, M., Solanki, S. K., Unruh, Y. C., Fröhlich, C., \& Wehrli, C. 1998, A\&A, 335, 709

Fröhlich, C. 2000, Space Sci. Rev., 94(1/2), 15

Fröhlich, C., \& Finsterle, W. 2001, VIRGO Radiometery and Total Solar Irradiance 1996-2000 revised, in Recent Insights into the Physics of the Sun and Heliosphere: Highlights from SoHO and other space missions, ed. P. Brekke, B. Fleck, \& J. B. Gurman, ASP Conf. Ser., IAU Symp., 203, 105
Fröhlich, C., \& Lean, J. 1998a, Total Solar Irradiance Variations, in New Eyes to see inside the Sun and Stars, ed. F. L. Deubner, et al., Proc. IAU Symp., 185, Kyoto, August 1997 (Kluwer), 89

Fröhlich, C., \& Lean, J. 1998b, Geophys. Res. Lett., 25, 4377

Gazis, P. R. 1996, Rev. Geophys., 34, 379

Harvey, K. L., \& Zwaan, C. 1993, Sol. Phys., 148, 85

Hoyt, D., \& Schatten, K. 1993, J. Geophys. Res., 98, 18, 895

Jokipii, J. R. 1991, Variations of the cosmic ray flux with time, in The Sun in Time, ed. C. P. Sonnet, M. S. Giampapa, \& M. S. Matthews (Univ. of Arizona Press), 205

Lean, J. 2000, Geophys. Res. Lett., 27 , 2425

Lean, J., Beer, J., \& Bradley, R. 1995, Geophys. Res. Lett., 22, 3195

Lockwood, M. 2001, J. Geophys. Res., 106, 16021

Lockwood, M., \& Stamper, R. 1999, Geophys. Res. Lett., 26, 2461

Lockwood, M., Stamper, R., \& Wild, M. N. 1999a, Nature, 399, 437

Lockwood, M., Stamper, R., Wild, M. N., Balogh, A., \& Jones, G. 1999b, Astron. \& Geophys, 40, 4.10

Mayaud, P. N. 1972, J. Geophys. Res., 77, 6870

McCracken, K. G., \& McDonald, F. B. 2001, The long-term modulation of the galactic cosmic radiation, 1500-2000, in Proc. 27th. Int. Cosmic Ray Conference, Hamburg, in press Moraal, H. 1993, Nucl. Phys. B, 33A, B, Proc. Suppl., 161

Potgieter, M. S. 1995, Adv. in Space Res, 16(9), 191

Pulkkinen, T. I., Nevanlinna, H., Pulkkinen, P. J., \& Lockwood, M. 2001, Space Sci. Rev., 95(1/2), 625

Rast, M. P., Fox, P. A., Lin, H., et al. 1999, Nature, 401, 678

Schatten, K. H., Wilcox, J. M., \& Ness, N. F. 1969, Sol. Phys., 6,442

Solanki, S. K., \& Fligge, M. 1998, Geophys. Res. Lett., 25, 341

Solanki, S. K., \& Fligge, M. 1999, Geophys. Res. Lett., 26, 2465

Solanki, S. K., Schüssler, M., \& Fligge, M. 2000, Nature, 480, 445

Solanki, S. K., Schüssler, M., \& Fligge, M. 2001, A\&A, in press Spruit, H. C. 1982, A\&A, 108, 356-360, 1982

Spruit, H. C. 1991, Theory of luminosity \& radius variations, in The Sun in Time, ed. C. P. Sonnet, M. S. Giampapa, \& M. S. Matthews (Univ. of Arizona Press), 118

Stamper, R., Lockwood, M., Wild, M. N., \& Clark, T. D. G. 1999, J. Geophys. Res., 104, 28, 325

Stuiver, M., \& Quay, P. D. 1980, Science, 207, 11

Suess, S. T., \& Smith, E. J. 1996, Geophys. Res. Lett., 23, 3267

Suess, S. T., Smith, E. J., Phillips, J., Goldstein, B. E., \& Nerney, S. 1996, A\&A, 316, 304

Usoskin, I. G., Kananen, H., Mursula, K., Tanskanen, P., \& Kovaltsov, G. A. 1998, J. Geophys. Res., 103, 9567

Wang, Y.-M., \& Sheeley, N. R. Jr. 1995, ApJ, 447, L143

Wang, Y.-M., Sheeley, N. R. Jr., \& Lean, J. 2000a, Geophys. Res. Lett., 27, 621

Wang, Y.-M., Sheeley, N. R. Jr., \& Lean, J. 2000b, Geophys. Res. Lett., 27, 505

Wilks, D. S. 1995, Statistical methods in the atmospheric sciences (Academic Press, San Diego)

Willson, R. C. 1997, Science, 277, 1963 